\title{
A Novel Approach towards Automatic Contour Identification of Jaw Cysts from Digital Panoramic Radiographs to improvise the Treatment planning
}

\author{
Veena Divya $\mathrm{K}^{1}$ \\ Dept. of Electronics \& Instrumentation Engineering, \\ RV College of Engineering, Bengaluru, 560059, India. \\ Email: veenadivya@gmail.com \\ Anand $\mathrm{Jatti}^{2}$ \\ Dept. of Electronics \& Instrumentation Engineering, \\ RV College of Engineering, Bengaluru, 560059, India. \\ Email: anandjatti@rvce.edu.in \\ Vidya .M.J ${ }^{3}$ \\ Director, Giritra Solutions, Nagarabhavi 2nd stage \\ Bengaluru, Karnataka, India \\ Email: vidyamjv@gmail.com \\ Revan Joshi ${ }^{4}$ \\ Radiologist, \\ Department of Oral Medicine and Radiology, \\ DA Pandu Memorial RV Dental College and Hospital, \\ Bengaluru, \\ India. \\ Email: revan.joshi@gmail.com \\ Srikar Gade ${ }^{5}$ \\ Department of Oral and Maxillofacial Surgery \\ Rajarajeswari Dental College \\ Ramohalli, Kumbalgodu, Mysore Road, Bangalore 560098 \\ Email: srikar0207@gmail.com
}

Received: June 10, 2021. Revised: November 1, 2021. Accepted: December 4, 2021. Published: January 3, 2022.

\begin{abstract}
Panoramic dental $x$-ray, a two-dimensional dental $x$-ray that captures the entire mouth in a single image, is used for the initial screening of various dental anomalies. One such is Jaw bone cyst, which, if not identified earlier, may lead to complications which in turn may lead to disfigurement and loss of function. Hence processing of radiographic images plays a vital role in identifying and locating the cystic region and extracting related features to assist clinical experts in further
\end{abstract}

analysis.

Objective: To develop an application of active contour model, known as Geodesic Active Contour, to generate Panoramic Dental X-Ray, a single $2 \mathrm{D}$ X-ray image of the entire mouth highlighting the dental specifications.

Methods: The process involves the image conversion from the OPG image into grayscale, Contrast adjustment using intensity level slicing, edge smoothing, segmentation, and cyst segmentation by Morphological Geodesic Active Contour to obtain the results. Hence processing of radiographic images plays a vital role in identifying and 
locating the cystic region. It is crucial in extracting related features to assist clinical experts in further analysis.

Conclusion: When efficient and accurate diagnostic methods exist, the treatment and cure become easy and concrete. Based on the morphological snake and level sets, it aims at identifying the boundary by minimizing the energy.

Results: Using the structural similarity index, an accuracy of $97.6 \%$ is obtained.

Advances in Knowledge: This process is advantageous as it is simpler, faster, and does not suffer from instability problems. Morphological methods improve their functional gradient descent by improving stability and speed. The hysteresis algorithm exhibits better edge detection performance, a significant reduction in computational time and scalability.

Keywords- Panoramic dental x-ray, OPG, Jaw bone cysts, Geodesic Active Contour.

\section{INTRODUCTION}

A cyst is a fluid-filled pathological cavity with a semipermeable membrane called epithelium (1). It is a prevalent pathology encountered in dentistry. They are formed around the root of the teeth or the buried teeth. If not detected early, cysts may result in the weakening of jaw bone and thus breaking it or the nearby teeth or other tissues in near proximity. Hence, early detection is essential for effective medical treatment. It also involves identifying the exact location of the cysts, the type, the area, and other related features for proper diagnosis and treatment planning. Figure 1 shows the presence of cyst in the oral cavity as depicted in a Panoramic X-Ray. Most cysts of the jaws are discovered incidentally on panoramic radiographs or when they adversely affect the surrounding structures and cause problems such as loosening of teeth or facial deformity (2). Panoramic radiographs or Orthopantomograms (OPG) are easy, cheaper and diagnosis can be performed as soon as image is taken. It is a significant and very commonly used imaging modality in dental practice.

It captures both jaw and supporting structures in a single image, i.e., the entire mouth. The cysts are characterized by low contrast, inhomogeneity, and blurred edges (1). Hence processing of the OPG images to segment the region of cystic lesion involves apt preprocessing techniques followed by a suitable segmentation algorithm.

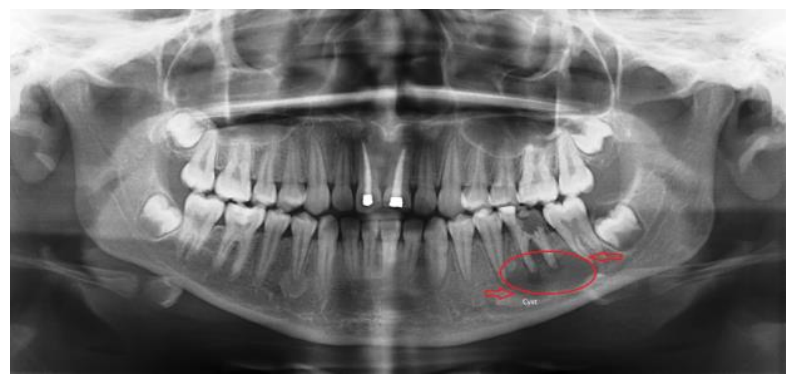

Fig. 1 A Panoramic X-Ray indicating a Cyst affecting the jaw and the teeth (Courtesy: R V Dental College)

Image segmentation is a process of grouping the pixels based on a predefined criterion. It is an inevitable step in any image processing application - segmentation results in dividing the image into several regions, which reflect meaningful information. The output of segmentation are image attributes which help in further analysis and interpretation.

The only available option in ensuring healthy years to life is an early diagnosis. The identification of a major part of the lesions in an early stage at dental clinics, this is done with the help of a routine radiography exam which is called orthopantomogram or panoramic radiograph. As a fact, the cystic lesion is more often identified as findings that are incidental on the panoramic radiographs, they have no symptoms that are apparent which is regardless about the chief complaint of the patient [13]. However, the requirement of an accurate interpretation has a training which can be challenging for even the professionals that are experienced. This is mainly caused because of the process of panoramic radiography. Wherein, the image that has been captured by sensors or the rotation of a plate around the head of the patient, this causes a superimposition of the structures that are bony in the facial skeleton.

Clinically, the early stages are symptomless, and it might cause an extensive destruction before the exposure of the radicular cyst. This may cause the affected area to expand. A periapical, well-circumscribed unilocular and radiolucency is seen on radiographs in the radicular cyst region. A cyst will cause the structures that are neighboring to be displaced, this is mainly in the mandibular canal, maxillary sinus, and root resorption has been occasionally seen. A follicular cyst and radicular cyst respectively are development of odontogenic cyst which causes a development around the crown of a tooth that is interrupted. Routine radiographs are mostly discovered when an eruption of a tooth has failed, teeth are tiled, or a tooth is missing. This is found most often in the age groups between the ages of 20 and 40 . In descending order, the teeth that are predisposed to impacted cysts are the third molar, second molar, and then first molar. the mandible is made up of $75 \%$ of the volume of the device [15].

There are basically three approaches: Boundary-based, Edgebased and Region based. The boundary-based approach involves locating the boundaries between the regions for the segmentation. The Edge-based approach is done through identifying the discontinuities and sudden intensity changes in the image that result in segmentation. In the Region-based segmentation, pixels are grouped into regions based on specific criteria (10). The selection of a proper segmentation approach is important in view to the above-mentioned OPG characteristics.

Geodesic Active contour model, an application of active contour model is implemented in this paper for segmentation of cystic region and hence extract the related features that assist clinical experts in further analysis and treatment planning. 


\section{LITERATURE SURVEY}

The following section discusses various other segmentation techniques used for the segmentation of cystic regions. J. Mikulka et al. (1) proposes level set, watershed, and the livewire segmentation method followed by evaluation of local descriptors like circularity, ratio of half axes of inscribed ellipse and area of cystic region for classification of the cysts. J. Mikulka et.al. (3) suggests a semiautomatic segmentation method using live-wire. It uses the shortest path algorithm for the demarcation of the area of interest. K. Anuradha et al. (4) achieves a segmentation method based on the markercontrolled watershed to segment cystic regions. Ingrid Nurtanio et al. (5) puts forward a method based on the application of active contour models for the segmentation of cysts. L. Lin et al. (6) describe a method to segment teeth based on analysis of local singularity analysis using Holder exponent. Rana et al. (7) propose the segmentation of cystic regions using Smartbrush, which is faster and more accurate than the threshold and manual segmentation methods. Pablo Márquez-Nelia et al. (8) establishes new results by connecting differential and morphological operators that provide stable and fast contour evolution using binary level set to improve processing time. Alsmadi et.al. (9) uses a hybrid Fuzzy C Means followed by Neutrosophic approach to segment cystic region in OPG. The performance and accuracy are assessed by determining area error metrics.

\section{METHODS AND MATERIALS}

The basic flow of methodology involves preprocessing followed by segmentation. OPG images are greatly affected due to various levels of noise produced by the imaging system, surrounding soft tissues, and also images suffer from low contrast. Hence, image preprocessing plays a vital role. Image preprocessing techniques are used to remove noise and enhance the visualization of an image and make it suitable for further processing. Medical image segmentation is an inevitable step for extracting disease features. Image segmentation is a process of dividing an image based on some criteria into various but meaningful objects. The segmentation results in raw pixels that correspond to either boundaries or regions $(10)(18)(19)(20)$. The main aim is to extract the area of interest with the required information. The algorithm of the proposed methodology is as shown below.

Algorithm:

STEP1: Image acquisition from the OPG image.

STEP2: Convert the image to grayscale.

STEP3: Contrast adjustment by Intensity level slicing.

STEP4: Edge smoothing refines edges and remove noise.

STEP5: Segmentation of mandibular region.

STEP6: Cyst segmentation by Morphological Geodesic

Active Contour to obtain the desired output

STEP7: Represent the obtained results using the Structural Similarity Index

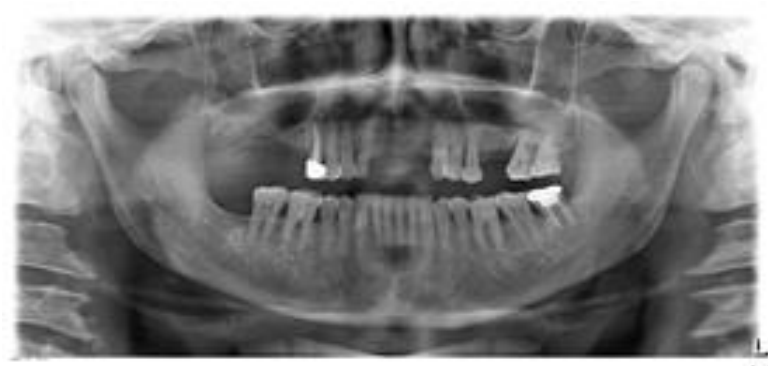

(a)

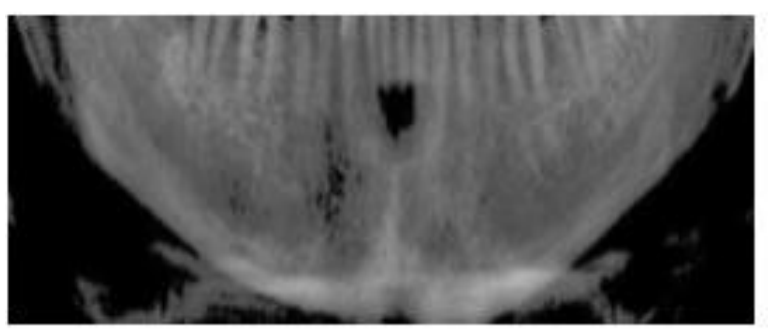

(b)

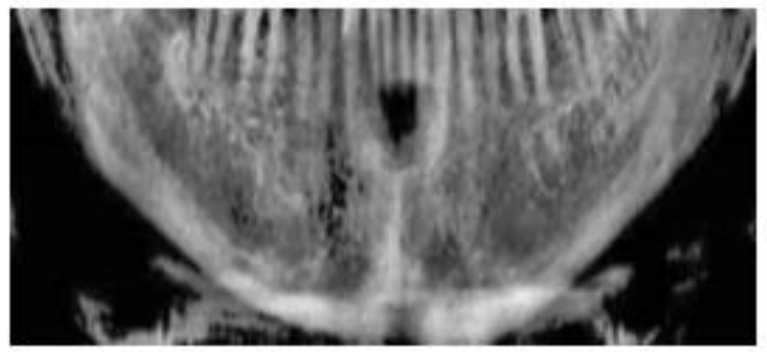

(c)

Fig. 2 (a) Input OPG image; (b) Post intensity level slicing; (c) Post edge smoothening

\section{A. Image Database}

A database containing 120 images showing different cysts has been used. The images were procured from DAPM RV Dental College and Hospital for the current research, and the ethical clearance for this work has been obtained with ref. No. DAPM RVDC / 182/18-19 dated: 07.05.2018. The OPG image is large-sized and suffers from low contrast and degradation due to various noise levels. The following section of the paper discusses the preprocessing and segmentation techniques applied on the OPG images for extraction of the cystic region.

\section{B. Image Preprocessing}

Panoramic images are usually degraded by the noise produced by imaging machines, surrounding soft tissues, and motion artifacts of jaws and tongues. The aim is to detect fine details and to enhance interpretability $(10)(18)(19)(22)$. The OPG images also suffer the presence of homogeneity surrounding the Region of Interest (ROI) and hence making the segmentation of ROI a problematic $\operatorname{task}(18)(22)$. A wise choice of preprocessing techniques is fundamental to improve the appearance of an image and enhance ROI for better visualization, interpretation, and analysis. Contrast adjustment 
is achieved by using Intensity Level Slicing. This results in summarizing all the pixel intensity corresponding to the cystic region to a single intensity value. This is followed by edge smoothening using a Gaussian filter. Figure 2(a) shows the input panoramic image, Figure 2(b) shows the image after intensity level slicing, and Figure 2(c) shows the image after edge smoothening.

The OPG images are large-sized, and hence, it is strenuous to find the cystic regions as the GAC method considers the whole image as the region of interest initially (20). In some cases, it may lead to the segmentation of teeth due to its higher intensity, thus resulting in improper segmentation. Therefore, to reduce implementation time and avoid segmentation of region that does not belong to the area of interest, the image is cropped.

\section{Image Segmentation}

ACTIVE contours or snakes have been used extensively in recent times, and it finds application widely in computer vision tools. They are mainly used for edge detection, contour detection, stereo matching, and other visual problems. They are extensively used for object boundary detection and tracking as well as segmenting 2D images. (8)(19)(20). An application of the active contour model is suggested for segmentation of cystic region in dental panoramic image in prior work in this field(19)(20). We use a morphological-based GAC for segmentation, as follows.

Let $\mathrm{C}: \mathrm{R}+\times(0,1) \rightarrow \mathrm{R}^{2}:(\mathrm{t}, \mathrm{p}) \rightarrow \mathrm{C}(\mathrm{t}, \mathrm{p})$ be a parametrized $2 \mathrm{D}$ curve over time. In the GAC framework, an energy functional, which depends on the contents of an image I, is assigned to a curve,

$$
\begin{aligned}
\mathrm{E}(\mathrm{C}) & =\int_{0}^{\text {length }(\mathrm{C})} \mathrm{g}(\mathrm{D})(\mathrm{C}(\mathrm{s})) \mathrm{ds} \\
& =\int_{0}^{1} \mathrm{~g}(\mathrm{D})(\mathrm{c}(\mathrm{p})) \cdot\left|\mathrm{c}_{\mathrm{p}}\right| \mathrm{dp}
\end{aligned}
$$

where $\mathrm{g}(\mathrm{I})$ is given by,

$$
\mathrm{g}(\mathrm{I})=\frac{1}{\sqrt{1+\alpha\left|\nabla \mathrm{G}_{\sigma} * \mathrm{I}\right|}}
$$

where Go* is a Gaussian filter with standard deviation $\sigma$.

The scalar field $\mathrm{F}$ deforms the curve $\mathrm{C}$ along with its average $\mathrm{N}$

$$
\mathrm{C}_{\mathrm{t}}=\mathrm{F} \cdot \mathrm{N}
$$

The steepest descent method is involved for minimization of the energy function defined in the equation,

$$
\mathrm{C}_{\mathrm{t}}=(\mathrm{g}(\mathrm{D}) \cdot \mathrm{K}-\nabla \mathrm{g}(\mathrm{D}) \cdot \mathrm{N}) \mathrm{N}
$$

Where $\mathrm{K}$ is the Euclidean curvature of $\mathrm{C}$ and with the given initial values $\mathrm{C}(0)=\mathrm{C}_{0}$.

Balloon force is used in cases where the attraction force is not strong enough for the evolution of the curve. The evolution with the auxiliary balloon force is

$\mathrm{C}_{\mathrm{t}}=(\mathrm{g}(\mathrm{I}) \mathrm{K}+\mathrm{g}(\mathrm{I}) \mathrm{v}-\nabla \mathrm{g}(\mathrm{I}) \cdot \mathrm{N}) \mathrm{N}$

The level set implementation of equation (5) is given as,

$$
\frac{\partial \mathrm{u}}{\partial \mathrm{t}}=\mathrm{g}(\mathrm{D})|\nabla \mathrm{u}| \operatorname{div}\left(\frac{\nabla \mathrm{u}}{|\nabla \mathrm{u}|}\right)+\mathrm{g}(\mathrm{D})|\nabla \mathrm{u}| \mathrm{v}+\nabla \mathrm{g}(\mathrm{I}) \nabla \mathrm{u}
$$

The above expression reflects three components. The smoothing force, balloon force, and image attraction force are responsible for bringing the hyper-surface to the exciting regions of the image. (8)

The smoothing force, which tends to smooth the hypersurface at high curvature segments given by,

$$
\frac{\partial \mathrm{u}}{\partial \mathrm{t}}=\mathrm{g}(\mathrm{I}) \cdot|\nabla \mathrm{u}| \operatorname{div}\left(\frac{\nabla \mathrm{u}}{|\nabla \mathrm{u}|}\right)
$$

where,

$$
\left.\mathrm{u}^{\mathrm{n}+1}(\mathrm{x})=\left((\underset{\mathrm{d}}{\operatorname{SI}} \underset{\widetilde{d}}{\operatorname{IS}})^{\mathrm{p}}\right) \mathrm{u}^{\mathrm{n}}\right)(\mathrm{x})
$$

The number of iterations of the algorithm of the above expression controls the success of the smoothening operator. This number is indicated by parameter $\mu \in \mathrm{N}$.

The balloon force, which inflates or deflates the hyper-surface in areas of little information.

$$
\frac{\partial \mathrm{u}}{\partial \mathrm{t}}=\mathrm{g}(\mathrm{I}) \cdot \mathrm{v} \cdot|\nabla \mathrm{u}|
$$

The strength of the balloon force is controlled by $g(\mathrm{I})$ and when $\mathrm{g}(\mathrm{I})$ is high, the curve is located far from a target region, and the balloon force must be strong; on the other hand, when $\mathrm{g}(\mathrm{I})$ becomes lower, the curve is approaching its objective, and hence the balloon force becomes unnecessary.

Hence, 


$$
\mathrm{u}^{\mathrm{n}+1}(\mathrm{x})=\left\{\begin{array}{ll}
\left(\mathrm{D}_{\mathrm{d}} \mathrm{u}^{\mathrm{n}}\right)(\mathrm{x}) & \text { if } \mathrm{g}(\mathrm{D})(\mathrm{x})>\theta \text { and } v>0 \\
\left(\mathrm{E}_{\mathrm{d}} \mathrm{u}^{\mathrm{n}}\right)(\mathrm{x}) & \text { if } \mathrm{g}(\mathrm{D})(\mathrm{x})>\theta \text { and } v<0 \\
\mathrm{u}^{\mathrm{n}}(\mathrm{x}) & \text { otherwise }
\end{array}\right\}
$$

(10) (8)

where Dd and Ed are the discrete versions of dilation and erosion and ' $u$ ' is the binary embedding function. The hypersurface is given as the level set 0.5 of a binary piecewise constant function $\mathrm{u}: \mathrm{Zd} \rightarrow\{0,1\}$. We take $\mathrm{u}(\mathbf{x})=1$ for every point $\mathbf{x}$ inside the hyper-surface, and $u(\mathbf{x})=0$ for every point $\mathbf{x}$ outside. The morphological operators will act on $u$ and, hence, they will implicitly evolve the hypersurface.

Hysteresis is the lagging effect. In the context of thresholding, for the areas above, a low threshold is considered if they are connected. Below, we compare morphological thresholding to hysteresis thresholding. This method ignores noise outside the edges. Since hysteresis thresholding is adopted for edge detection, two thresholds (high and low) need to be computed.

A finely quantized gradient magnitude histogram is used to find the high and low hysteresis thresholds. For calculating the high threshold, a non-uniform quantizer is implemented. For the medium edge, strong edge, and edge/texture block, the most significant peak in the gradient magnitude histogram of the input image falls near the origin, indicating that the lowintensity pixels form the maximum in number. The edge pixels form a series of smaller peaks, in which each peak represents edges having similar gradient magnitudes (8). So, the high threshold needs to be selected between the highest and the second-highest peak, which is most likely an edge peak. The quantizer should have more quantization levels in the area between the highest peak and the second highest peak and fewer levels in other parts. The highest and lowest values of the gradient magnitudes in the block considered are averaged to get the first reconstruction level (R1).

The second reconstruction level (R2) is obtained as the mean of the first reconstruction level and the most negligible value of the gradient magnitude. Similarly, $\mathrm{n}$ reconstruction levels are computed. The corresponding equations are given below.

$\mathrm{R} 1=($ least + most $) / 2$

$\mathrm{Ri}+1=($ least $+\mathrm{Ri}) / 2$ for $(\mathrm{i}=1,2,3, \ldots 7)$

Where least and most are the lowest and highest values of the gradient magnitudes, respectively. Here the histogram is divided into 8 reconstruction levels ( $\mathrm{R} 1$ to $\mathrm{R} 8$ ). Now, compare the total number of pixels in each reconstruction level $\mathrm{Ri}$ (NoPixels_Ri) with the product of P1 value and the total number of pixels in the block (NoPixels P1) to choose the Reconstruction level i, where NoPixels_Ri is nearest to the NoPixels_P1. Using the selected level Ri and highest gradient magnitude, mag_most, the high threshold ThHigh is computed.

The low threshold ThLow is calculated as $40 \%$ of the high threshold (20). Here the selected reconstruction level $\mathrm{Ri}$ is considered as a threshold factor, Thresh_Factor. The proposed novel computation of a high threshold is given below. Low threshold calculation has been adapted from (8).

ThHigh $=(($ mag_most + Thresh_Factor $) / 2)$

ThLow $=$ ThHigh $\times 0.4$

(14)(8)

Finally, hysteresis thresholding is performed on each block using these calculated high and low thresholds, ThHigh and ThLow, respectively to obtain the required edge image.

The accuracy of the obtained results is established by the structural similarity index. The Structural Similarity Index (SSIM) is a perceptual metric that quantifies image quality degradation caused by processing such as data compression or by losses in data transmission. It is an indication of how similar the processed image is to the original image.

\section{RESULTS AND DISCUSSION}

The proposed model is implemented in Python OpenCV. The projected method segments the cystic regions effectively. The marking of the region of cysts helps create a safety margin.

The OPG image is converted to grayscale and is cropped to eliminate irrelevant regions. Further, it is subjected to segmentation by Geodesic Active contour and hysteresis segmentation to extract the region of interest. The algorithm was applied on a set of 89 images showing different types of cysts. Area and perimeter can be used in the preparation of surgeries.Solidity value is used to measure the scope of cyst for diagnosis of cysts, it can be used to estimate the stages of the stages of the disease.

Study of the solidity of cysts such as roundness and circularity in relation to the form descriptors (such as the size of the perimeter and the ball) was made to determine Consider cysts that and allow the term "oblong" to be applied to things with the lines of measurement drawing near to 1 to be cylindrical. On the basis of these results, it can be concluded that radicular cysts are elliptical, with an approximate ellipse centric diameter of 0.871 , and follicular cysts are generally oblong, with a diameter of 0.45 .

Intensities, modal and mean modal greys, and aspect ratio for cyst regions can be critical in classifying cysts.

These findings have been shown to provide more useful and pertinent details for the follow-up diagnosis and future planning use of OPG images.

Table 1. Accuracy achieved and implementation time for 89 different OPG images

\begin{tabular}{|l|l|l|}
\hline NAME & TIME(s) & ACCURACY \\
\hline 1 & 193.76 & 99.05 \\
\hline 2 & 46.8 & 95.08 \\
\hline
\end{tabular}




\begin{tabular}{|l|l|l|}
\hline 3 & 266.04 & 99.36 \\
\hline 4 & 227.92 & 99.25 \\
\hline 5 & 195.49 & 98.22 \\
\hline 6 & 201.73 & 98.82 \\
\hline 7 & 45.1 & 90.78 \\
\hline 8 & 227 & 99.53 \\
\hline 9 & 173.31 & 98.99 \\
\hline 10 & 178.62 & 98.86 \\
\hline
\end{tabular}

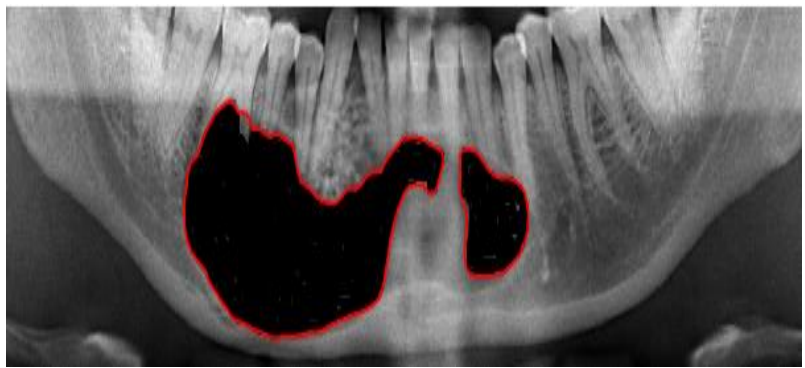

(b)

Fig. 4 Segmentation of cystic regions using geodesic active contour model shown on different panoramic images (a) \& (b).

Table 2. depicts the performance analysis for ten images among the segmented data set.

\section{Performance Analysis}

\section{Percentage Accuracy \& Time in Seconds}

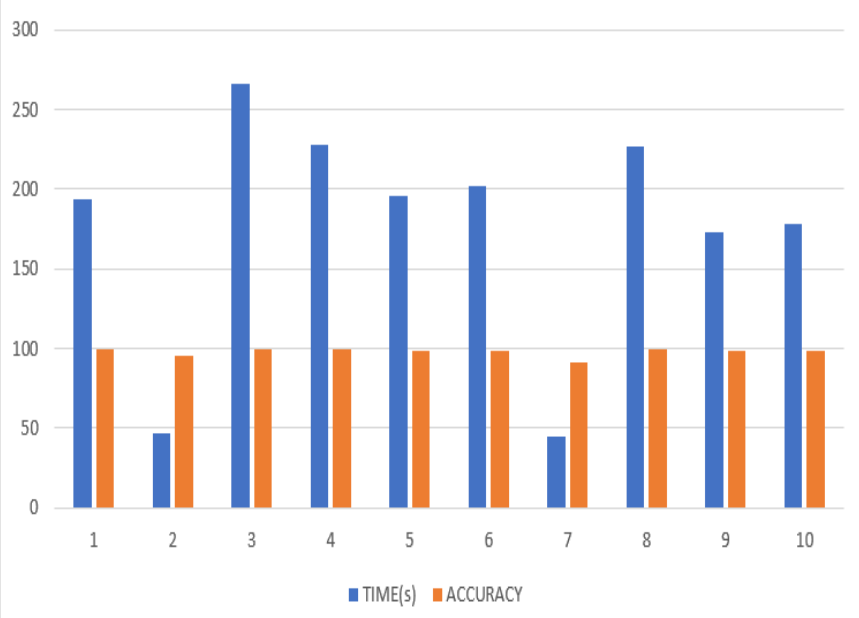

Fig. 3 Plot of Percentage accuracy and time is taken to process images.

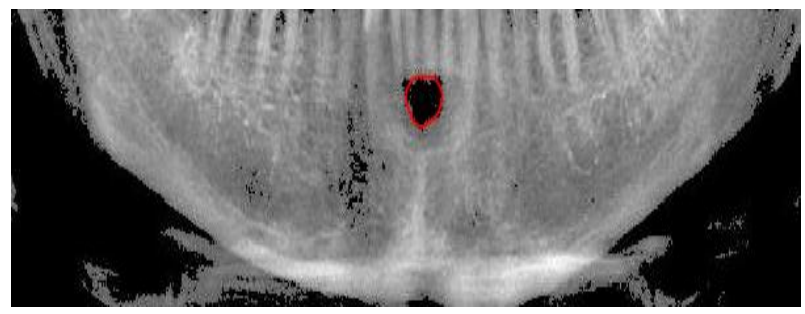

(a)

\begin{tabular}{|c|c|c|c|c|c|c|c|c|c|c|c|}
\hline \multirow{2}{*}{ Patient name } & \multirow{2}{*}{$\begin{array}{l}\text { actual pirel } \\
\text { area }\end{array}$} & \multirow{2}{*}{$\begin{array}{l}\text { cyst pisel } \\
\text { arean }\end{array}$} & \multirow{2}{*}{ Entropy } & \multicolumn{2}{|c|}{ Contrast } & \multicolumn{2}{|c|}{ Correlation } & \multicolumn{2}{|c|}{ Enengy } & \multicolumn{2}{|c|}{ Homogeneity } \\
\hline & & & & $\min$ & $\max$ & $\min$ & $\max$ & $\min$ & max & min & $\max$ \\
\hline Image 1 & 77312 & 3043 & 6.7931106052 & 0.2778 & 0.289 & 0.936 & 0.9365 & 0.1111 & 0.123 & 0.876 & 0.8887 \\
\hline Image ? & 80668 & 13666 & 6.1110503329 & 0.2076 & 0.2616 & 0.9251 & 0.9404 & 0.2173 & 0.2246 & 0.9051 & 0.9172 \\
\hline Image 3 & 801668 & 15730 & 5.82492965 & 0.4386 & 0.5437 & 0.9216 & 0.9365 & 0.163 & 0.1672 & 0.8624 & 0.8743 \\
\hline Image 4 & 90,450 & 2802 & 7.12251451818 & 0.2807 & 0.3009 & 0.924 & 0.93 & 0.1286 & 0.1346 & 0.8704 & 0.8889 \\
\hline Image 5 & 89847 & 3937 & 6.815565664 & 0.261 & 0.2786 & 0.9079 & 0.926 & 0.1661 & 0.1711 & 0.8939 & 0.9081 \\
\hline Image 6 & 82611 & 9591 & 0.6958602805 & 0.3039 & 0.4816 & 0.922 & 0.9515 & 0.0969 & 0.1057 & 0.8566 & 0.88811 \\
\hline Image 7 & 89998 & 5858 & 6.692296144 & 0.2931 & 0.3085 & 0.9204 & 0.9239 & 0.1503 & 0.1508 & 0.8938 & 0.8939 \\
\hline Image 8 & 64628 & 12999 & 59327\%4231 & 0.942 & 0.5516 & 0.8727 & 0.9363 & 0.1558 & 0.1564 & 0.8584 & 0.8757 \\
\hline Image 9 & 79728 & 946 & 7.097867359 & 0.3198 & 0,3315 & 0.9263 & 0.9288 & 0.106 & 0.11112 & 0.8666 & 0.8785 \\
\hline Image 10 & 81270 & 2280 & 6.660999951 & 0.2314 & 0.3187 & 0.8947 & 0.9258 & 0.1626 & 0.1635 & 0.8967 & 0.904 \\
\hline
\end{tabular}

Table 3. Accuracy achieved and implementation time for ten different OPG images for hysteresis segmentation.

\begin{tabular}{|l|l|l|}
\hline NAME & TIME(s) & $\begin{array}{l}\text { ACCURACY } \\
(\%)\end{array}$ \\
\hline 1 & 30 & 90.05 \\
\hline 2 & 46 & 95.08 \\
\hline 3 & 26 & 92.36 \\
\hline 4 & 22 & 96.25 \\
\hline 5 & 19 & 90.22 \\
\hline 6 & 20 & 92.82 \\
\hline 7 & 45 & 90.78 \\
\hline 8 & 22 & 95.53 \\
\hline 9 & 17 & 92.99 \\
\hline 10 & 18 & 93.66 \\
\hline
\end{tabular}


Table 2 shows the accuracy achieved for each of the images and the implementation time. The average accuracy attained is $92.9 \%$.
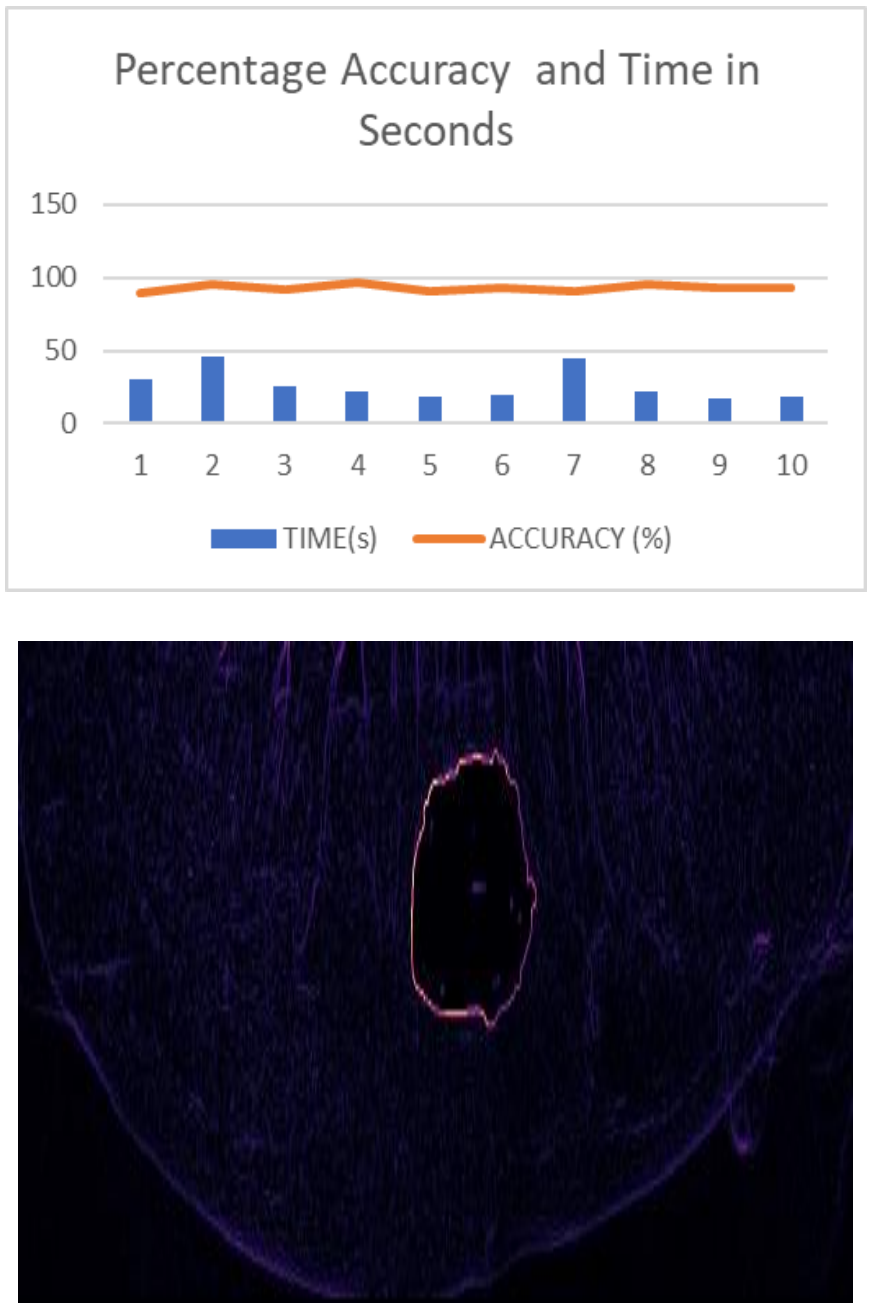

(a)

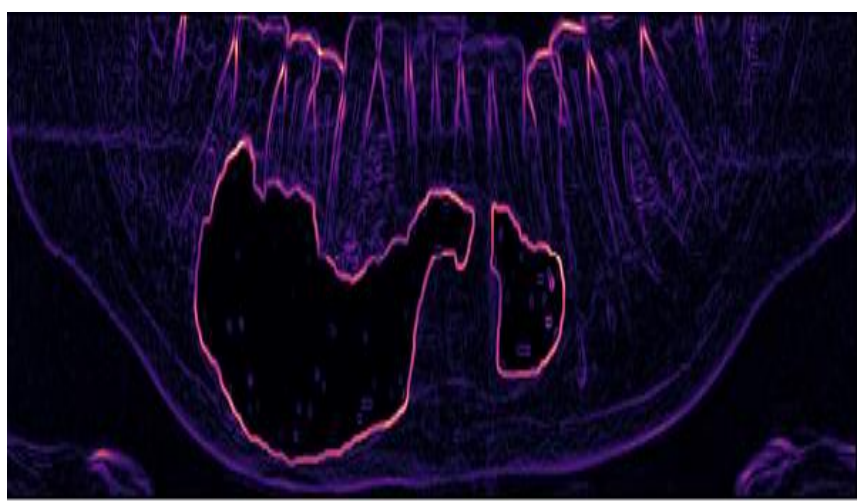

(b)

Fig. 5 Segmentation of cystic regions using semantic segmentation shown on the panoramic images (a) \& (b).

\section{CONCLUSION}

This paper gives a morphological-based geodesic active contour algorithm for segmentation of cystic regions in panoramic images. This process is advantageous as it is simpler, faster, and does not suffer from instability problems. Figure 3 depicts the percentage of accuracy. Figures 4 and 5 give a clear identification of the cystic regions that are improved in terms of accuracy and the proven the processing time is much lesser than the previous work(20).

This method does not require re-initialization of the embedded function ' $u$ ' is to the fact that the morphological method is being used. Morphological methods improve their functional gradient descent by improving stability and speed. The hysteresis algorithm exhibits better edge detection performance, a significant reduction in computational time and scalability. A combinational method could be developed for further improvement in accuracy, stability and speed in the future.

Image interpretation of dental X-rays is a difficult task, and the research community pays little attention to it. In addition, a new taxonomy concentrating on imaging modalities-based categorization such as bitewing, periapical, panoramic, $\mathrm{CBCT} / \mathrm{CT}$, hybrid datasets and colour photos is also being developed. According to various research papers, the variety of image datasets makes selecting a single segmentation strategy challenging when using standard image processing methods. According to numerous academics cited in this paper, annotated datasets are the key roadblock in the development of a high-performance classification algorithm. Because of the unusual image, dental X-ray imaging data is not the same as other medical images.

\section{References}

[1] Mikulka J, Kabrda M, Gescheidtova E, Perina V. Classification of jawbone cysts via orthopantomogram processing. 2012 35th International Conference on Telecommunications and Signal Processing (TSP). 2012;

[2] Stoetzer M, Nickel F, Rana M, Lemound J, Wenzel D, von See $\mathrm{C}$ et al. Advances in assessing the volume of odontogenic cysts and tumors in the mandible: a retrospective clinical trial. Head \& Face Medicine. 2013;9(1).

[3] Proceedings, Conference Proceedings, IEEE Papers | Proceedings.com [Internet]. Proceedings.com. 2019 [cited 24 November 2019]. Available from: http://www.proceedings.com/Progress in Electromagnetics Research Symposium

[4] Anuradha K, Sankaranarayanan K. Detection of Oral Tumor based on Marker Controlled Watershed Algorithm. International Journal of Computer Applications. 2012;52(2):15-18.

[5] Nurtanio I, Purnama I, Hariadi M, Purnomo M. Cyst and Tumor Lesion Segmentation on Dental Panoramic Images using Active Contour Models. IPTEK The Journal for Technology and Science. 2011;22(3). 
[6] Lin P, Huang P, Huang P. An effective teeth segmentation method for dental periapical radiographs based on local singularity. International Conference on System Science and Engineering (ICSSE). 2013;

[7] Rana M, Modrow D, Keuchel J, Chui C, Rana M, Wagner $\mathrm{M}$ et al. Development and evaluation of an automatic tumor segmentation tool: A comparison between automatic, semi-automatic and manual segmentation of mandibular odontogenic cysts and tumors. Journal of Cranio-Maxillofacial Surgery. 2015;43(3):355-359.

[8] Marquez-Neila P, Baumela L, Alvarez L. A Morphological Approach to Curvature-Based Evolution of Curves and Surfaces. IEEE Transactions on Pattern Analysis and Machine Intelligence. 2014;36(1):2-17.

[9] Alsmadi M. A hybrid Fuzzy C-Means and Neutrosophic for jaw lesions segmentation. Ain Shams Engineering Journal. 2018;9(4):697-706.

[10] Gonzalez R, Woods R. Digital image processing. New Delhi: Dorling Kindersley; 2014.

[11] Shear M, Speight P, Shear M. Cysts of the oral and maxillofacial regions. Oxford: Blackwell Munksgaard; 2007.

[12] Nirmala Devi S, Kumaravel N. Comparison of active contour models for image segmentation in X-ray coronary angiogram images. Journal of Medical Engineering \& Technology. 2008;32(5):408-418.

[13] Kaushik A, C Mathpal P, Sharma V. Edge Detection and Level Set Active Contour Model for the Segmentation of Cavity Present in Dental X-Ray Images. International Journal of Computer Applications. 2014;96(9):24-29.

[14] Abdallah Y, Boshara M. Assessment of field size on radiotherapy machines using texture analysis. Sudan Medical Monitor. 2014;9(1):5.

[15] Johnson N, Gannon O, Savage N, Batstone M. Frequency of odontogenic cysts and tumors: a systematic review. Journal of Investigative and Clinical Dentistry. 2013;5(1):9-14.

[16] Ulaganathan G, Banumathi A, Amutha J, Jeevani Selvabala A. Dental cyst delineation using live wire algorithm. 2012 International Conference on Machine Vision and Image Processing (MVIP). 2012;

[17] Veena Divya K, Jatti A, Joshi R, Krishna S. A study and analysis of image enhancement techniques augmenting dental pantamograms to review jawbone cysts and tumors. TENCON 2017 - 2017 IEEE Region 10 Conference. 2017; .

[18] Veena Divya K., Jatti A, Joshi R, Deepu Krishna S. Characterization of dental pathologies using digital panoramic X-ray images based on texture analysis. 2017 39th Annual International Conference of the IEEE Engineering in Medicine and Biology Society (EMBC). 2017;

[19] Veena Divya K, Jatti A, Joshi R, meharaj S. 2016 IEEE EMBS Conference on Biomedical Engineering and Sciences (IECBES). [S.l.]: IEEE; 2016.

[20] Veena Divya K., Jatti A, Sabah Meharaj P, Joshi R. Image processing and parameter extraction of digital panoramic dental X-rays with ImageJ. 2016 International Conference on Computation System and Information Technology for Sustainable Solutions (CSITSS). 2016;.

[21] Rafeek Mamdouh, Hazem M. El-Bakry, Alaa Riad, Nashaat El-Khamisy, Converting 2D-Medical Image Files "DICOM" into 3D- Models, based on Image Processing, and Analysing their Results with Python Programming, WSEAS Transactions on Computers, ISSN / E-ISSN: 1109-2750 / 2224-2872, Volume 19, 2020, Art. \#2, pp. $10-20$.

\section{Contribution of Individual Authors to the Creation of a Scientific Article (Ghostwriting Policy)}

Veena Divya Krishnappa carried out the algorithm implementation and the optimization.

Dr. Anand Jatti has designed the results and simulation of the entire work.

Dr. Vidya M.J has worked on the python programming part of the simulation.

Dr.Revan Kumar Joshi and Dr. Srikar Gade, are involved in the validation of the results obtained to guide and also responsible for writing part of the paper.

\section{Sources of Funding for Research Presented in a Scientific Article or Scientific Article Itself}

No funding is utilized for the research carried out, but it is done under ethical clearance between RV College of Engineering and DA Pandu memorial RV dental College and Hospital, Bengaluru.

\section{Creative Commons Attribution License 4.0 (Attribution 4.0 International, CC BY 4.0)}

This article is published under the terms of the Creative Commons Attribution License 4.0

https://creativecommons.org/licenses/by/4.0/deed.en_US 